\title{
Development and psychometric testing of nurses' professional commitment inventory
}

\author{
Fateme Jafaraghaee ${ }^{1}$, Abbas Ebadi ${ }^{2,3}$, Shadi Dehghanzadeh ${ }^{4}$, Neda Mehrdad ${ }^{5,6} *$ (D) \\ Received: 3 Aug 2020 \\ Published: 30 Sep 2021
}

\begin{abstract}
Background: Promotion of nurses' professional commitment is one of the strategies for retaining nurses and preventing their turnover. The aim of this study was the development and psychometric testing of the Nurses' Professional Commitment Inventory.

Methods: This mixed method study was performed in an item generation and a psychometric testing phase. In the first phase, a 34item inventory was developed based on the results of a grounded theory and the existing literature. Search date was 2010 to May 2018. In the second phase, we recruited 272 clinical nurses and tested the psychometric properties of the inventory. Construct validity was tested via the exploratory factor analysis. Reliability testing was performed through test-retest stability and internal consistency testing. SPSS version 21.0 (SPPS Corp) was used for statistical analysis. Significance level was set at $\mathrm{p}<0.05$.

Results: In the first phase, a 74-item pull was extracted. After reviewing, the primary version of the Nurses' Professional Commitment Inventory (NPCI) with 34 items was developed. Eight items were deleted during psychometric testing. In factor analysis, the remaining 26 items were loaded on 3 factors, namely professional attachment, professional performance, and internalization of the profession. These factors explained $53.92 \%$ of the total variance of professional commitment. The Cronbach's alpha and mean testretest intraclass correlation coefficient for NPCI were 0.92 and 0.88 , respectively.

Conclusion: The Nurses' Professional Commitment Inventory has acceptable validity and reliability. This inventory includes dimensions that indicate the formation of professional commitment. The items of the scale can reveal nurses' strengths and weaknesses related to professional commitment.
\end{abstract}

Keywords: Instrument development, Nurse, Professional commitment, Psychometric testing

Conflicts of Interest: None declared

Funding: The study is supported financially by THE research deputy of Tehran University of Medical Sciences.

*This work has been published under CC BY-NC-SA 1.0 license.

Copyright $\odot$ Iran University of Medical Sciences

Cite this article as: Jafaraghaee F, Ebadi A, Dehghanzadeh Sh, Mehrdad N. Development and psychometric testing of nurses' professional commitment inventory. Med J Islam Repub Iran. 2021 (30 Sep);35:127. https://doi.org/10.47176/mjiri.35.127

\section{Introduction}

Nursing staff shortage has been a global nursing challenge in the last 2 decades (1-3). Yet the number of nurses who leave their profession is still increasing $(4,5)$. A study reported that nurses' intention to leave nursing increases by five times from the first to the fifth year of their nursing practice (6). This results in major damages to both nursing education and workforce management systems.

Corresponding author: Dr Neda Mehrdad, nmehrdad@tums.ac.ir

1. School of Nursing and Midwifery, Guilan University of Medical Sciences, Rasht, Iran

2. Behavioral Sciences Research Center, Life Style Institute, Faculty of Nursing, Baqiyatallah University of Medical Sciences, Tehran, Iran

3. Nursing Faculty, Baqiyatallah University of Medical Sciences, Tehran, Iran

4. Department of Nursing, College of Nursing and Midwifery, Rasht Branch, Islamic Azad University, Rasht, Iran

5. Endocrinology and Metabolism Research Center, Endocrinology and Metabolism Clinical Sciences Institute, Tehran University of Medical Sciences, Tehran, Iran

6. Nursing care Research Center, Iran University of Medical Sciences, Tehran, Iran
Thus, nursing managers attempt to use strategies to retain more nurses (7).

Professional commitment $\left(\mathrm{P}^{\prime} \mathrm{C}\right)$ promotion is one of the most important strategies for retaining nurses and preventing their turnover (8). PC is defined as a belief in professional goals and values, willingness to make significant efforts on behalf of the profession, and desire to remain

$\uparrow$ What is "already known" in this topic:

To the best of our knowledge, the only nursing-specific professional commitment measurement instrument is the Nurses' Professional Commitment Scale developed in Taiwan.

$\rightarrow$ What this article adds:

Nurses' Professional Commitment Inventory is a valid and reliable questionnaire. It has acceptable generalizability because it was developed through both empirical data collected from hospital nurses and data retrieved from the existing literature. 
part of the profession (9). It is also considered as emotional attachment to the profession (10) and the degree of belief in professional values and goals and identity development based on the profession $(11,12)$. Career-related aspects of PC have turned it into an important construct in vocational psychology and career development. Individuals with stronger PC are more interested in their profession and make more serious attempt for career development (13).

The concept of PC is close to the concept of organizational commitment, so that they are sometimes used interchangeably. However, while PC refers to one's attitude toward profession and acceptance of professional values and goals (14), organizational commitment is considered as loyalty to the organization and intention to stay in it (15). Consequently, PC is far beyond organizational commitment (11). Other concepts similar to PC are occupational commitment and career commitment. These 2 concepts are used to refer to the motivation for working in a certain occupation (16). These concepts are also interchangeably used with PC $(13,12)$.

PC has many different outcomes. It determines the degree to which employees value their professions $(13,11)$, the level of their professional efficacy, intention to stay in their professions (8), and job satisfaction (17). Employees with deeper PC are more interested in their professions, have greater desire for professional contribution and development $(13,11)$, establish stronger relationships with other professions, attract greater respect and attention to their own professions, and attempt to challenge and broaden their professional skills (4). Similarly, nurses with deeper PC are more committed to provide quality care and develop their profession $(18,14)$. Deep PC is also associated with nurses' more effective communication with patients (19), greater patient safety, better patient outcomes (9), higher job satisfaction (18), and lower negative emotions (8).

Given the significant effects of PC on different aspects of nurses' professional practice, its measurement is also of great importance in nursing (20). Therefore, numerous studies have been conducted $(21,14,11)$ and a wide range of instruments have been used for its measurement. In some studies, researcher-made PC measurement instruments were developed based on the existing literature (22, $21,19)$. In some other studies, PC was measured through instruments developed based on mixed-method designs for the measurement of organizational and occupational commitment $(11,12)$ and professional identity $(23,24)$. Table 1 presents a list of available instruments for PC measurement.

A review of these instruments reveals that most of them measure PC in professions other than nursing (14, 25, 26 ), and hence may not be directly and readily applicable to the unique conditions of nursing as a profession, which directly deals with human life, health, and well-being. For example, Tansakul et al (27) developed a professional commitment scale, which specifically measures PC among teachers. Moreover, some of PC measurement instruments are not profession-specific; rather, they are used for PC measurement in different professions, irrespective of their unique characteristics and conditions. Among these broad instruments are Blau's Career Commitment Scale (28) and Carson and Bedeian's Career Commitment Measure (29).

To the best of our knowledge, the only nursing-specific PC measurement instrument is the Nurses' Professional Commitment Scale developed by Lin et al in Taiwan. This scale contains 19 items in the 3 main domains: (1) nursing professional compliance; (2) involvement of nursing professionals; and (3) retention of nursing professionals (30). This scale was developed based on the existing literature and nurses' clinical experiences, and its developers noted that it is appropriate for PC measurement among Taiwanese nurses (30). Working context in different cultures can affect PC (9). Therefore, this study was done based on a comprehensive inductive and deductive approach to address these gaps. The aim of the study was the development and psychometric testing of Nurses' Professional Commitment Inventory (NPCI).

Table 1. The characteristics of the existing $\mathrm{PC}^{1}$ measurement instruments

\begin{tabular}{|c|c|c|c|c|c|}
\hline Instrument title & Developers & Source & $\begin{array}{l}\text { Number of } \\
\text { items }\end{array}$ & $\begin{array}{l}\text { Cronbach's } \\
\text { alpha }\end{array}$ & $\begin{array}{l}\text { Psychometric testing } \\
\text { Methods }\end{array}$ \\
\hline $\begin{array}{l}\text { Occupational Commitment } \\
\text { Measure }\end{array}$ & $\begin{array}{l}\text { Meyer et al. } \\
\text { (1993) }\end{array}$ & $\begin{array}{c}\text { Meyer and Allen's Model of } \\
\text { Commitment }\end{array}$ & $\begin{array}{c}18 \text { items in } \\
\text { three domains }\end{array}$ & $0.7-0.8$ & $\begin{array}{c}\text { Confirmatory factor } \\
\text { analysis }\end{array}$ \\
\hline Career Commitment Scale & Blau (1985) & Existing literature & 7 Items & 0.91 & $\begin{array}{c}\text { Exploratory factor } \\
\text { analysis } \\
\text { Convergent validity } \\
\text { Discriminant validity }\end{array}$ \\
\hline $\begin{array}{l}\text { Career Commitment Meas- } \\
\text { ure }\end{array}$ & $\begin{array}{l}\text { Carson and } \\
\text { Bedeian } \\
(1994)\end{array}$ & Existing instruments & $\begin{array}{l}24 \text { items in } \\
\text { three domains }\end{array}$ & $0.8-0.9$ & $\begin{array}{l}\text { Convergent validity } \\
\text { Discriminant validity } \\
\text { Construct validity }\end{array}$ \\
\hline $\begin{array}{l}\text { Teachers' Professional } \\
\text { Commitment }\end{array}$ & $\begin{array}{l}\text { Tansakul et al. } \\
\text { (2015) }\end{array}$ & $\begin{array}{l}\text { Meyer's Organizational Commit- } \\
\text { ment Measure and interviews }\end{array}$ & $\begin{array}{l}18 \text { items in } \\
\text { three domains }\end{array}$ & $0.8-0.9$ & $\begin{array}{c}\text { Content validity } \\
\text { Differential item func- } \\
\text { tioning } \\
\text { Exploratory factor } \\
\text { analysis } \\
\text { Confirmatory factor } \\
\text { analysis }\end{array}$ \\
\hline $\begin{array}{l}\text { Nurses' Professional Com- } \\
\text { mitment Scale }\end{array}$ & $\begin{array}{l}\text { Lin et al. } \\
(2007)\end{array}$ & Existing literature and instruments & $\begin{array}{l}19 \text { Items in } \\
\text { three domains }\end{array}$ & $0.69-0.93$ & $\begin{array}{c}\text { Content validity } \\
\text { Principle component } \\
\text { analysis }\end{array}$ \\
\hline
\end{tabular}

\footnotetext{
Professional Commitment
} 


\section{Methods}

This mixed method study was conducted in an NPCI item generation phase and an NPCI item reduction phase.

\section{Phase I: NPCI Item Generation}

One of the approaches to item generation is the inductive-deductive approach in which necessary data are collected through a qualitative study and supplemented through a literature review (31). The items of NPCI were generated using this approach and based on the existing literature and the results of a grounded theory into the process of PC formation among hospital nurses (32). The grounded theory study was conducted as the first author's $\mathrm{PhD}$ dissertation. The data for that study were collected via in-depth semi-structured interviews with 21 hospital nurses in different private and public teaching and nonteaching hospitals in different cities in Iran.

Sampling was started purposefully with maximum variation and continued theoretically up to the point of data saturation. As theoretical sampling, also 2 nurse supervisors and 1 matron were interviewed. Data were managed using the MAXQDA 10 software (VERBI). Data analysis through open, axial, and selective coding (33) resulted in the development of 974 primary codes, which were categorized into 38 subcategories and 4 main categories. The 4 main categories were self-encounter, interaction with the organization and the profession, promotion of the professional role, and attachment to the profession. The first category involves the nurse's primary understanding of nursing as a profession based on his/her conditions and the decision for staying in or leaving the profession. The second category, that is, interaction with the organization and the profession, includes organizational and professional facilitators and barriers to the formation of PC. The third main category, that is, promotion of the professional role, refers to the attempt the nurse makes to highlight his/her role and presence in the profession. PC is the outcome of the nurse's actions so that the nurse is so engaged in his/her profession that considers it as part of his/her own identity and makes any attempt to develop his/her profession. These 4 main categories were considered as the primary domains of NPCI, and subsequently 63 items on nurses' PC were generated using the results of the grounded theory study.

In order to develop a comprehensive instrument with greater generalizability, we also performed a literature search to retrieve studies into PC measurement. Search key terms were organizational commitment, career commitment, occupational commitment, professional commitment, scale, tool, and questionnaire. Literature search was conducted in online databases, including CINAHL, PubMed, British Nursing Index, EMBASE, and PsycINFO from 2010 to May 2018. Inclusion criteria were publication in English peer-reviewed journals and easy access to document full-texts.

Retrieved studies were assessed for eligibility and 17 eligible studies were reviewed. Appropriate items for NPCI were extracted from the reviewed studies. Moreover, the items of all existing PC-related instruments were docu- mented. Finally, 11 new items were added to the already generated 63 items, and a pool with 74 items was generated. A panel of experts carefully reviewed and compared these 74 items to select the most appropriate items for PC assessment. The criterion for selecting the most appropriate items was maximum agreement among the members of the panel. Repetitive, overlapping, or inappropriate items were revised or removed and an inventory with 34 items was developed. The 34-item inventory was then subjected to psychometric testing.

\section{Phase II: NPCI Item Reduction (Psychometric Testing)}

According to instrument development scholars, the best individuals for assessing the relevance of an instrument to a given concept are its actual users. In other words, involvement of the users of an instrument in the process of its psychometric evaluation can increase its relevance and quality (34). Therefore, the face validity of NPCI was assessed by the potential users, nurses. Face validity was tested both qualitatively and quantitatively. For qualitative testing of face validity, face-to-face interviews were held with 10 hospital nurses to assess the difficulty, ambiguity, and appropriateness of the items. Nurses were purposively selected from different wards of 2 public hospitals. Difficult or ambiguous items for the nurses were revised and clarified. Quantitative face validity of the inventory was tested by asking 15 hospital nurses to rate the importance of the items on a 5-point scale as follows: "very important" (scored 5), "important" (scored 4), "relatively important" (scored 3), "slightly important" (scored 2), and "not important" (scored 1). Then, item impact score was calculated via the "Importance $\times$ Frequency $(\%)$ " formula. The minimum acceptable impact score was 1.5 (35).

Content validity of NPCI was assessed through calculating content validity ratio (CVR) and content validity index (CVI). At least 3 experts are needed for content validity assessment, although more experts can provide more reliable data (36). Therefore, for calculating CVR, 15 nursing instructors and hospital nurses with expertise in instrument development or psychometric evaluation were purposively selected. They were provided with information about the study aim, the theoretical and practical definitions of the NPCI domains, and a copy of the inventory, and were asked over email to rate the necessity of the items on the following 3-point scale, "necessary, "useful but not necessary", and "unnecessary". Then, CVR was calculated. The minimum acceptable CVR was 0.60 (35).

For CVI calculation, the same experts were asked over email to rate the relevance of NPCI items on a 4-point scale as "irrelevant" (scored 1), "needs revision" (scored 2), "relatively relevant" (scored 3), and "completely relevant" (scored 4) (31). Twelve experts sent their rating responses. Finally, item CVI (I- CVI) was calculated via dividing the number of experts rated that item 3 or 4 by the number of experts. The minimum acceptable I- CVI value 0.80 (36). Thus, items with CVI less than 0.80 were deleted.

Construct validity of NPCI was tested through exploratory factor analysis. The 2 main goals of factor analysis are to reduce the data and clarify the structure of the in- 
tended construct or theory (31). Factor analysis in the present study was conducted to determine the dimensions of $\mathrm{PC}$ and to determine the placement of each item in the determined dimensions. Sample size was estimated based on 10 participants per item rule (36). A total of 300 nurses were asked to fill out NPCI. They were conveniently recruited from 6 hospitals (including 3 public teaching hospitals, a private hospital, a semi-private hospital, and 1 military hospital) in Rasht and Tehran, Iran. Inclusion criteria were employment in hospital settings as staff nurse, head-nurse, or nurse supervisor; and clinical work experience of more than 1 year. Participants filled out NPCI on a self-report basis.

Construct validity of NPCI was tested through exploratory factor analysis. The collected data were used for exploratory factor analysis with varimax rotation. KaiserMeyer-Olkin test was run to test sampling adequacy and Bartlett's test was run to test the appropriateness of the factor analysis model. The number of factors was determined using scree plot and eigenvalues. The minimum acceptable factor loading was 0.4 (31).

Another method for NPCI construct validity testing was to test its convergent validity. Meyer and colleagues' Occupational Commitment Measure was determined to be appropriate for testing the convergent validity of NPCI because the occupational commitment concept has close similarities to the PC concept, while they are not exactly the same. Accordingly, 100 nurses were conveniently recruited from the study setting to complete Meyer and colleagues' Occupational Commitment Measure (37) in addition to NPCI. Then, Pearson correlation analysis was conducted for assessing the correlation between these 2 instruments.

Internal consistency was tested by calculating Cronbach's alpha. Alpha values more than 0.7 are interpreted as good internal consistency (36). Moreover, testretest stability was used for reliability testing. Accordingly, 30 nurses were conveniently recruited from the study setting to twice complete NPCI with a 14-day period in between. Test-retest intraclass correlation coefficient (ICC) was calculated. ICC values higher than 0.7 are interpreted as good test-retest stability (31).

Ethical considerations in all the study stages have been mentioned. This study has the approval of the ethics committee of Tehran University of Medical Sciences, Tehran, Iran (code: 92-02-99-23464-136280). We gave all participants explanations about the study aim, the voluntary nature of participation in the study, the anonymity of the data collection instruments, and the exclusive use of the collected data for the present study. Written informed consent was gotten from all participants. The protocol of study has been published (38).

\section{Results}

The primary version of NPCI contained 34 items. The CVR values of all items were more than 0.60 . However, 2 items obtained impact scores of less than 1.5 and 5 items obtained CVIs of less than 0.83 . These 7 items were deleted. The 27-item NPCI was then subjected to construct validity testing.

\begin{tabular}{|c|c|c|c|}
\hline \multicolumn{2}{|l|}{ Characteristics } & \multirow{2}{*}{$\begin{array}{c}\text { Number } \\
185\end{array}$} & \multirow{2}{*}{$\begin{array}{c}\text { Percent } \\
68\end{array}$} \\
\hline Gender & Female & & \\
\hline & Male & 87 & 32 \\
\hline \multirow[t]{3}{*}{ Marital status } & Single & 77 & 28 \\
\hline & Married & 192 & 71 \\
\hline & Widowed & 3 & 1 \\
\hline \multirow[t]{2}{*}{ Nursing degree } & Bachelor's & 248 & 91 \\
\hline & Master's & 24 & 9 \\
\hline \multirow{7}{*}{$\begin{array}{l}\text { Affiliated hospi- } \\
\text { tal ward }\end{array}$} & Medical & 74 & 27 \\
\hline & Surgical & 57 & 21 \\
\hline & Critical care & 91 & 33 \\
\hline & Emergency & 32 & 12 \\
\hline & Pediatric & 8 & 3 \\
\hline & Psychiatric & 5 & 2 \\
\hline & Nursing office & 5 & 2 \\
\hline \multirow[t]{4}{*}{ Work shift } & Fixed/Morning & 79 & 29 \\
\hline & Fixed/Evening & 12 & 4 \\
\hline & Fixed/Night & 21 & 8 \\
\hline & Rotational & 160 & 59 \\
\hline \multirow{3}{*}{$\begin{array}{l}\text { Organizational } \\
\text { position }\end{array}$} & Registered nurse & 227 & 83 \\
\hline & Head nurse & 35 & 13 \\
\hline & Supervisor & 10 & 4 \\
\hline Total & & 272 & 100 \\
\hline
\end{tabular}

Among 300 nurses who were recruited to answer NPCI, 272 returned their inventories completely filled out. Table 2 demonstrates their characteristics.

The value of Kaiser-Meyer-Olkin test was 0.92 and the result of Bartlett's sphericity test was statistically significant $(\mathrm{p}<0.001)$. Thus, study sample was adequate; there were significant correlations between the items, and the items were factorable. Factor analysis with varimax rotation revealed that NPCI had 3 main factors. Eigenvalues and scree plot also showed the appropriateness of the 3factor structure of the inventory. The extracted 3 factors were labeled as professional attachment, professional performance, and internalization of the profession. These 3 factors explained $53.92 \%$ of the total variance of PC (Table 3). One item had a factor loading value of less than 0.40 , and hence was deleted. The remaining 26 items were loaded on the 3 factors.

The correlation coefficients between the mean scores of all extracted 3 factors and the total score of NPCI ranged from 0.85 to $0.92(\mathrm{p}<0.05)$. Moreover, the mean score of NPCI had a significant correlation with the mean score of Meyer and colleagues' Occupational Commitment Measure, with a correlation coefficient of 0.435 .

The Cronbach's alpha value of NPCI was 0.92 and the test-retest ICC was 0.88 , both confirming the acceptable reliability of the inventory (Table 4 ).

\section{Discussion}

In this study, NPCI was developed and its psychometric properties were tested. NPCI items were generated based on the results of a grounded theory (32) and the existing literature. The final NPCI contained 26 items in the 3 domains of professional attachment, professional performance, and internalization of the profession.

The first domain of NPCI, that is, professional attachment, contained 14 items. The highest percentage of the explained variance in this domain was related to the item, "I have stayed in the profession at personal will and inter- 
Table 3. Factor loading values of the items and the percentage of variance explained by each factor

\begin{tabular}{|c|c|c|c|}
\hline Items & Factor I & Factor II & Factor III \\
\hline 3. I have stayed in the profession at personal will and interest. & 0.787 & & \\
\hline 21. I encourage my relatives/friends to enter this profession. & 0.651 & & \\
\hline 6. I look for an opportunity to leave this profession. & -0.608 & & \\
\hline 20. I encourage my colleagues to join professional nursing organizations. & 0.590 & & \\
\hline 1. I chose this profession with personal interest. & 0.573 & & \\
\hline 10. I like to have more responsibilities in this profession. & 0.562 & & \\
\hline 4. I have accepted my role as a nurse. & 0.508 & & \\
\hline 5. I feel responsibility to stay in this profession. & 0.492 & & \\
\hline 9. I like to continue my education to be able to develop my profession. & 0.474 & & \\
\hline 7. I feel I work in an important profession. & 0.465 & & \\
\hline 8. I feel compelled to strive in this profession. & 0.450 & & \\
\hline 19. I am a member of a professional nursing organization. & 0.429 & & \\
\hline 17. I follow the news about the profession. & 0.398 & & \\
\hline 18. Events in the profession are important to me. & 0.303 & & \\
\hline 16. As a professional, I empathize with the patient. & & 0.731 & \\
\hline 15. As a professional, I attempt to establish the best relationship with the patient. & & 0.703 & \\
\hline 14. I do my best to provide better care. & & 0.661 & \\
\hline 13. I like to update my professional knowledge. & & 0.601 & \\
\hline 12. I am well-known among my colleagues as a professional role model. & & 0.482 & \\
\hline 11. I fulfill my professional responsibilities in the best manner. & & 0.463 & \\
\hline 23. I show my abilities in front of colleagues and other healthcare professionals. & & & 0.773 \\
\hline 24. I like other healthcare providers to have good attitudes towards my profession. & & & 0.753 \\
\hline 25. I defend my profession in front of others. & & & 0.711 \\
\hline 22. I protect the credit of my profession. & & & 0.705 \\
\hline 26. The profession of nursing has been internalized for me. & & & 0.628 \\
\hline 27. In any circle, I proudly introduce myself as a nurse. & & & 0.625 \\
\hline The percentage of the explained variance & 19.086 & 18.423 & 16.413 \\
\hline
\end{tabular}

\begin{tabular}{lccc} 
Table 4. The Cronbach's alpha and the $\mathrm{ICC}^{1}$ values of $\mathrm{NPCI}^{2}$ and its domains & & \\
\hline Domains & Number of Items & Cronbach's alpha & ICC (95\% confidence intervals) \\
\hline Professional attachment & 14 & 0.814 & $0.898(0.780-0.953)$ \\
Professional performance & 6 & 0.868 & $0.743(0.425-0.883)$ \\
Internalization of the profession & 6 & 0.900 & $0.724(0.398-0.873)$ \\
Total NPCI & 26 & 0.920 & $0.882(0.747-0.945)$ \\
\hline
\end{tabular}

1. Inter Class Correlation

2. Nurses' Professional Commitment Inventory

est." PC is an internal characteristic, which is affected by personal motivations. It includes individuals' emotional commitment to stay in their professions (39). The Nurses' Professional Commitment Scale also includes similar items as the items of the professional attachment domain of NPCI (30).
The second domain of NPCI, that is, professional performance, included 6 items. The greatest variance in this domain was related to the item, "As a professional, I empathize with the patient." It is noteworthy that nursing performance is mainly viewed in relation to patient care (40), and hence empathy with patient is considered as a 
determinant of PC (30).

The third domain of NPCI was internalization of the profession and included 6 items. The greatest variance in this domain was related to the item, "I show my abilities in front of colleagues and other healthcare professionals." This item denotes the desire for introducing the profession to other professions. Health care professionals' unrealistic understanding of nursing practice and its knowledge base has caused them to give limited importance to nursing (41). Nurses' ability to introduce their professional abilities to other people is effective in developing their profession, and hence can be considered as an indicator of nurses' commitment to professional development.

Some items of the professional attachment and the internalization of the profession domains of NPCI are in some ways similar to 2 domains of affective commitment and normative commitment in Occupational Commitment Measure (37). Moreover, the items in the professional attachment and the internalization of the profession domains of NPCI were similar to the items of the career planning and career identity domains of the Career Commitment Measure (29). However, NPCI is different from the Career Commitment Measure because it mainly focuses on commitment to a profession rather than on commitment to solely clinical practice or a career.

Psychometric testing of NPCI was performed using different methods. Convergent validity testing revealed significant correlations between the scores of NPCI domains and the total score of the inventory. Moreover, it showed that NPCI score was moderately correlated with the score of the Occupational Commitment Measure. It is noteworthy that the correlation coefficient in convergent validity testing should not be close to 1; rather, a moderate correlation coefficient is preferable and shows great convergent validity (31). Reliability testing also revealed the great stability of NPCI over time and its great internal consistency.

There is one limitation in our study. Participants' selfreport answering to NPCI might have affected the correlations among its items. However, we attempted to collect more reliable data through ensuring participants that their data would be managed confidentially.

\section{Conclusion}

In this study, NPCI was developed with 26 items in 3 main domains. NPCI has acceptable validity and reliability for PC measurement instrument. Answering to its items and to the 12 items on nurses' demographic characteristics takes only 15 minutes. Thus, NPCI can be used as a valid and reliable easy-to-use instrument for PC measurement among hospital nurses. It probably has acceptable generalizability because it was developed through both empirical data collected from hospital nurses and data retrieved from the existing literature.

\section{Acknowledgement}

The authors acknowledge all the nurses who participated in the study and all the experts whose opinions we used in determining the face and content validity of the inventory. Also, we acknowledge the research deputy of Tehran
University of Medical Sciences for their financial support of the study.

\section{Conflict of Interests}

The authors declare that they have no competing interests.

\section{References}

1. Marc M, Bartosiewicz A, Burzyńska J, Chmiel Z, Januszewicz P. Anursing shortage: a prospect of global an local policies. Int Nurs Rev. 2019;66:9-16

2. Nantsupawat A, Kunaviktikul W, Nantsupawat R, Wichaikhum OA, Thienthong H, Poghosyan L. effects of nurse work environment on job disssactisfaction, burnout, intention to leave. Int Nurs Rev. 2017;64:91-8.

3. Yu F, Raphael D, Mackay L, Smith M, King A. pPersonal and workrelated factors associated with nurse resiliance: A systematic review. Int J Nurs Stud. 2019;93:129-40.

4. Bakker EJM, Kox JHAM, Miedema HS, Bierma-Zeinstra S, Runhaar J, Boot CRL, et al. Physical and mental determinants of droupout and retention amoung nursing students: protocol of the SPRING cohort study. BMC Nurs. 2018:17-27.

5. Lu H, Ahao Y, While A. Job satsfaction among hospital nurses. Int $\mathbf{J}$ Nurs Stud. 2019;94:21-31.

6. Labrague LJ, McEnroe- Petiiie DM, Tsaras K, Cruz JP, Colet PC, Gloe DS. Organisational commitment and turnover intention among rural nurses in the philippines: implications for nursing management. Int J Nurs Sci. 2018;5:403-8.

7. Zhu J, Rodgers S, Melia KM. Underestanding human resources wastage in the nursing shortage: lesson learned from Chines nurses leaving nursing practice. Athens J Health. 2018;5(3):195-212.

8. Hoeve WT, Brouwer J, Kunnen S. Turnover prevention: the direct and indirect assocoation between organizational job stressors, negative emotions and professional commitment in novice nurses. $\mathrm{J}$ Adv Nurs. 2020;76:836-45.

9. Garcia-Moyano L, Altisent R, Pellicer-Garcia B, Guerrero-Portilla S, Arrazola-Albedi O, Delgado-Marroquin MT. A concept analysis of professional commitment in nursing. Nurs Ethics. 2017;26:778-97.

10. Akbari O, Vagharseyyedin SA, Farajzadeh Z. The Relationship of organizational justice with nurses' professional commitment. Modern Care J. 2015;12(1):31-8.

11. Lewicka D. Interpersonal Trust at Work and Organisational and Professional Commitment Interdependency Model. J Pos Manag. 2015;6(3):83-100.

12. Zheng J, Wu G. Work-family conflict, perceived organizational support and professional commitment: A mediation mechanism for Chinese project professionals. Int J Environ Res Public Health. 2018;15(2):344-65

13. Jiang Z. Emotional intelligence and career decision-making selfefficacy: Mediating roles of goal commitment and professional commitment. J Employ Couns. 2016;53(1):30-47.

14. Clements AJ, Kinman G, Leggetter, S, Teoh K, Guppy A. Exploring commitment, professional identity, and support for student nurses. Nurse Educ Pract. 2016;16(1):20-6.

15. Setyowati R, Priyotomo S. Investigating organizational commitment among medical doctors, hospital nurses and two other professional jobs: a syatematic review. Glob J health Sci. 2017;9(12):99-106.

16. Wilson ME, Liddell DL, Hirschy AS, Pasquesi K. Professional identity, career commitment, and career entrenchment of midlevel student affairs professionals. J Coll iStud Dev. 2016;57(5):557-72.

17. Hegazy HMH, Aqylalmazamir H. Relationship between professional commitment, job satisfaction and professionl roles practice level of medical social workers. J Sociol Soc Work. 2017;5(2):139-50.

18. Barac I, Prlic N, Pluzaric J, Farcic N, Kovacecic S. The mediating role of nurses professional commitment in the relationship betweencore self self-evaluation and job satisfaction. Int J Occup Med Environ Health. 2018;31(5):1-10.

19. Siraneh Y, Ololo S, Tsega G, Yitbarek K, Adamu A, Erchafo B, et al. Level and Factors Associated with Professional Commitment of Health Professionals Providing Institutional Delivery Services in Public Health Facilities, Southwest Ethiopia. Ethiop J Health Sci. 2018;28:495-504.

20. Schmidt BJ, Mcarthur EC. Professional nursing values: A concept 
analysis. Nurs Forum. 2018;53:67-5.

21. Caricati L, Sollami A, Bianconcini M, Guidi C, Prandi C, Silvano R, et al. The role of professional and team commitments in nursephysician collaboration. J Nurs Manag. 2016;24(2):E192-E200.

22. Barutcu CD, Ergin S. Examination of Professional Commitment and Stress Management among Nurses from Different Generations. Int J Caring Sci. 2017;10(1):456-463.

23. Panari C, Caricati L, Pelosi A, Rossi C. Emotional exhaustion among healthcare professionals: the effects of role ambiguity, work engagement and professional commitment. Acta Biomed Health Prof. 2019;90:60-6.

24. Dwivedula R, Bredillet CN, Muller R. Personality and work motivation as determinants of project success: the mediating role of organizational and professional commitment. Int $\mathrm{J}$ Manag Dev. 2016;1(3):229-44.

25. Mufitha MB, Teng LS, Chen, YC. Is professional commitment a reason for turnover intention in professionals? Adv Bus Int J. 2019:124-43.

26. Nugroho AP, Hariyanto AT. The role of Job characteristics and profesional commitment to tenure and performance( study of teachers in public high schools in Solo raya. J Indones Sci Echon Res. 2019;1(1):26-31.

27. Tansakul J, Kamata A, Patarapichayatham C, Kanjanawasee S. An Evaluation of Items and Dimensional Structure of a Scale to Measure Teachers' Professional Commitment in Thailand. Asian Soc Sci. 2015;11(16):15-24.

28. Blau GJ. The measurement and prediction of career commitment. J Occup Psycho. 1985;58(4):277-88.

29. Carson KD, Bedeian AG. Career commitment: Construction of a measure and examination of its psychometric properties. J Vocat Behav. 1994:44(3):237-62.

30. Lin CJ, Wang HC, Li TC, Huang LC. Reliability and Validity of Nurses job satisfaction and nurses professional commitment scale. Mid-Taiwan J Med. 2007;12(2):65-75.

31. Waltz CF, Strickland OL, Lenz ER. Measurement in nursing and health research. 5th edition. Springer publishing company; 2017.

32. Jafaragaee F, Mehrdad N, Parvizy S. A model of professional commitmentM formation in clinical nurses [dissertation] 2012. Nursing and Midwifery school: Tehran University of Medical Sciences. 2012 (In Persian).

33. Strauss A, Corbin, J. Basics of Qualitative Research technics and procedures for developing Grounded Theory. 2 nd ed. Sage Publication; 1998.

34. Connell J, Carlton J, Grundy A, Buck ET, Keetharuth AD, Ricketts $\mathrm{T}$, et al. The importance of content and face validity in instrument development: lesson learnt from service users when developing the Recovering Quality of Life measure (ReQol). Qual Life Res. 2018;27:1893-902.

35. Ebadi A, Zarshenash L, Zareian A, Sharifnia H, Mojahedi M. Principles of scale development in health sciences. Tehran: Jame-eNegar Publishing house; 2017. (In Persian).

36. Polit DF, Beck CT. Nursing Research Generating and Assessing Evidence for Nursing practice 10 th ed. Wolters Kluwer; 2017.

37. Meyer JP, Allen NJ, Smith CA. Commitment to organizations and occupations: Extension and test of a three-component conceptualization. J Appl Psychol. 1993;78(4):538- 51.

38. Jafaraghaee F, Ebadi A, Negarande, R, Mehrdad N. A professional commitment scale foe clinical nurses a protocol study. Med J Islam Repub Iran. 2017(23 Dec);31.123.

39. Mitchell R, Boyle B, Stieglitz SV. Professional commitment and team effectiveness: A modrated mediation investigation of cognitive diversity and task conflict. J Bus Psychol. 2019;34:471-83.

40. Chiang YC, Lee HC, Chu TL, Han CY, Hsiao YC. The impact of nurses spiritual health on theire attitudes toward spiritual care, professional commitment, and caring. Nurs Outlook. 2016;64:215-24.

41. Hoeve Y, Janson G, Roodbol P. The nursing profession: public image, self-concept and professional identity. A discussion paper. J Adv Nurs. 2014;70(2):295-302. 